AECG criteria and ACR/EULAR criteria, respectively. SGUS revealed SS-like changes in $37 / 64$ patients (59\%); interestingly, SS-like findings were observed in 22/23 patients in the European cohort, compared to 15/40 patients in the Brazilian cohort.

Conclusions: Common symptoms and findings in jSS include dry mouth, systemic manifestations and salivary gland enlargement, followed by reduced tear secretion and hyposalivation.

Disclosure of Interest: None declared

DOI: 10.1136/annrheumdis-2017-eular.5804

\section{SAT0271 IS THERE A NEED TO INCLUDE SEROLOGICAL PATTERN TO PREDICT DAMAGE IN PATIENTS WITH ANTIPHOSPHOLIPID SYNDROME: DIAPS APPLICATION}

D. Mazilu $^{1}$, D. Potarniche ${ }^{2}$, I. Saulescu ${ }^{1}$, A. Borangiu ${ }^{1}$, L. Groseanu ${ }^{1}$, C. Constantinescu ${ }^{1}$, V. Vlad ${ }^{2}$, D. Opris ${ }^{3}$, A. Balanescu ${ }^{1}$, D. Predeteanu ${ }^{1}$, R. Ionescu 1. " "Sfanta Maria" Clinical Hospital, "Carol Davila" University of Medicine; ${ }^{2}$ "Sfanta Maria" Clinical Hospital; " "Sfanta Maria" Clinical Hospital, "Carol Davila" Univeristy of Medicine, Bucharest, Romania

Background: Antiphospholipid syndrome (APS) is an autoimmune disease defined as the presence of antiphospholipid antibodies (aPL), at least a clinical thrombotic event and is associated with an important risk of organ damage. The new index proposed, Damage Index in patients with Thrombotic Antiphospholipid Syndrome (DIAPS) may be an useful tool to estimate cumulative damage in patients with primary and secondary APS. It includes 38 clinical items expanded to show the complexity of clinical manifestations in APS patients.

Objectives: The aim of this study is to analyze the serological pattern as potential predictive factor for an increased DIAPS.

Methods: All consecutive patients known with APS according to the Sapporo and/or Sydney classification criteria were included in our monocentric cohort. Data on medical history, clinical manifestations, aPL profile and medication were collected. DIAPS score was used to measure damage in each patient. The relationship between aPL profile and DIAPS score was analysed.

Results: Seventy six patients with APS were included: 11 patients with primary APS, 65 patients with secondary APS. Their mean disease duration was $9.59 \pm 7.39$ years. The most frequent clinical manifestation from DIAPS was the peripheral vascular (deep vein thrombosis, intermittent claudication, tissue loss, vascular venous insufficiency) found in $61.8 \%$ of patients, followed by the neuropsychiatric manifestations $(46.1 \%)$. The mean DIAPS score in our cohort was $4.25 \pm 3.51$, not significantly different between patients with primary vs secondary APS (4.72 vs $4.16, p=0.629$ ). Lupus anticoagulant (LA) was found in 25 patiens $(32.9 \%)$, anti cardiolipin antibodies (aCL) in 49 patients $(64.5 \%)$ and antibodies to $\beta 2$-glycoprotein I ( $\beta 2 \mathrm{GPI})$ in 23 patients $(30.3 \%)$. There were 36 patients known with a single positive aPL (47.4\%), 27 patients $(35.5 \%)$ with 2 positive aPL and only 2 patients with triple positivity. There were no significant differences regarding antibody profile between patients with primary and secondary APS. Higher values of DIAPS were seen in patients with $\beta 2 G P I$ $(p=0.042)$ and with positivity for $2 \mathrm{aPL}(p=0.003)$. DIAPS value correlated to the presence of $\beta$ 2GPI ( $p=0.042, R=0.233$ ) and to positivity for two aPL ( $p=0.003$, $\mathrm{R}=0.341$ ).

Conclusions: Our study suggests that double positivity for aPL, especially the presence of $\beta 2 \mathrm{GPI}$ confers an increased value of DIAPS in patients with primary and secondary APS.

References:

[1] M-C Amigo et al. Development and initial validation of a damage index (DIAPS) in patients with thrombotic antiphospholipid syndrome (APS). Lupus (2015) 24, 927-934.

[2] LM Amezcua-Guerra. Improving definitions for an index of cumulative organ damage in patients with the antiphospholipid syndrome (DIAPS). Lupus (2016) $25,671-672$.

Disclosure of Interest: None declared

DOI: 10.1136/annrheumdis-2017-eular.4105

\section{SAT0272 LACK OF ASSOCIATION OF GLUCOCORTICOID EXPOSURE AND METABOLIC SYNDROME IN SLE}

\section{Apostolopoulos, A. Hoi, E. Morand. Monash Health, Melbourne, Australia}

Background: The Metabolic Syndrome (MetS) is a disorder of energy utilisation and storage, associated with an increased risk of cardiovascular (CV) disease. MetS may contribute to the increased CV disease in SLE, but the prevalence, cause, and impact of MetS in SLE is poorly understood, as are the effects of glucocorticoid (GC) exposure.

Objectives: To characterise the prevalence of the features of MetS in a wellcharacterised cohort of SLE patients, and determine the effect of GC use on these parameters.

Methods: SLE patients studied as part of a single centre prospective longitudinal cohort. Disease activity (SLEDAI-2K), treatment and laboratory details were recorded at each visit. Other investigation results were collected from institution databases. MetS defined as $\geq 3$ criteria $^{1}: \mathrm{BMI}>30 \mathrm{~kg} / \mathrm{m}^{2}$; triglycerides $>1.7 \mathrm{mmol} / \mathrm{L}$; $\mathrm{HDL}$-cholesterol $<1.3 \mathrm{mmol} / \mathrm{L}$; blood pressure $>130 / 85 \mathrm{mmHg}$ or treatment for hypertension; fasting glucose $>5.6 \mathrm{mmol} / \mathrm{L}$ or treatment for hyperglycaemia.
Continuous variables were described as median (IQR), and compared using Kruskal-Wallis tests. Categorical variables were described as frequency and compared using Chi-squared tests.

Results:

\begin{tabular}{lcccc}
\hline & Total (289) & GC exposed (211) & GC not -exposed (78) & p-value \\
\hline BMI $>30 \mathrm{~kg} / \mathrm{m}^{2}$ & $50(17 \%)$ & $33(24 \%)$ & $17(22 \%)$ & 0.22 \\
éTriglycerides & $80(28 \%)$ & $69(33 \%)$ & $11(14 \%)$ & 0.002 \\
êHDL-cholesterol & $95(33 \%)$ & $73(35 \%)$ & $22(28 \%)$ & 0.33 \\
é Fasting glucose & $32(11 \%)$ & $24(11 \%)$ & $8(10 \%)$ & 1.00 \\
Hypertension & $137(47 \%)$ & $110(52 \%)$ & $27(35 \%)$ & 0.01 \\
Metabolic Syndrome & $49(17 \%)$ & $36(17 \%)$ & $13(17 \%)$ & 1.00 \\
\hline
\end{tabular}

289 patients were included (87\% female; 51\% Caucasian, 29\% Asian), and median age at enrolment of 37.7y. Median follow-up was 3.43y (med 15 visits). Time adjusted-mean SLEDAI (AMS) over the study period was 3.67. 81\% (211) patients received GC (time-adjusted mean $4.25 \mathrm{mg}$ prednisolone/d) and AMS was significantly higher in GC-exposed patients (4.19 vs 1.97 [EM1], $\mathrm{p}<0.01$ ). MetS criteria were met by $49(17 \%)$ of patients (Table 1). Hypertriglyceridaemia and hypertension were significantly more frequent in GC-treated patients, but the prevalence of obesity and other MetS domains, or MetS overall, were not. There were significantly more patients with MetS score $=0$ in the GC-exposed subset $(43 / 78$ vs $76 / 211 p<0.01)$.

The prevalence of obesity of $17 \%$ is lower than in the general population. There was no significant change in BMI across the period of observation and surprisingly, no association between GC exposure and change in BMI.

Conclusions: The prevalence of MetS in SLE was lower than previously reported in other, smaller, lupus cohorts ${ }^{2,3}$. This study suggests GC exposure was associated with hypertriglyceridaemia and hypertension in SLE. Potential negative effects of active disease on MetS domains require further investigation. References:

[1] Alberti KGMM, Eckel RH, Grundy SM, et al. Harmonizing the metabolic syndrome: a joint interim statement of the International Diabetes Federation Task Force on Epidemiology and Prevention; National Heart, Lung, and Blood; AHA; WHF; International Atherosclerosis Society; International Association for the Study of Obesity. Circ 2009;120(16):1640-45.

[2] Parker B, Ahmad Y, Shelmerdine J, et al. An analysis of the metabolic syndrome phenotype in systemic lupus erythematosus. Lupus 2011;20(14):1459-65.

[3] Chung CP, Avalos I, Oeser A, et al. High prevalence of the metabolic syndrome in patients with systemic lupus erythematosus: association with disease characteristics and cardiovascular risk factors. ARD 2007;66(2):20814.

Disclosure of Interest: None declared

DOI: 10.1136/annrheumdis-2017-eular.6764

\section{SAT0273 FACTORS RELATED TO ALEXITHYMIA IN SYSTEMIC LUPUS ERYTHEMATOSUS}

D.P.E. Margiotta, F. Basta, M. Vadacca, G. Dolcini, M. Lo Vullo, A. Rigon, A. Afeltra. Unit of Allergology, Immunology and Rheumatology, Università Campus Bio-Medico di Roma, Rome, Italy, Rome, Italy

Background: Several evidences described a considerable prevalence of alexithymia among patients with chronic diseases, such as systemic autoimmune diseases. In patients affected by Systemic Lupus Erythematosus (SLE), alexithymia seems to be related to mood disorders and personality.

Objectives: In this study we evaluated alexithymia in relation to HR-QoL (Health related Quality of Life) and to factor associated to HR-QoL, such as mood disorders, fatigue, work ability, sleep quality and physical activity.

Methods: We consecutively enrolled SLE patients and healthy controls in a cross sectional study with a retrospective design. We wvaluated alexithymia by the Toronto Alexithymia Scale 20 (TAS-20). AHR-QoL was expressed by MOS-SF-36. Mood disorders was assessed by BDI and HAM-H. Fatigue was evaluated by Facit-Fatigue. Physical activity was quantified using International Physical Activity Questionnaire (IPAQ) and the sleep quality using the Pittsburgh Sleep Quality Index (PSQI). Work ability was assessed by Work Productivity and Activity Impairment (WPAI). Cognitive impairment was defined according to MOCA screening test.

Results: Fifty-two SLE patients and 50 age-matched healthy subjects were enrolled in the study. Mean TAS-20 score was significantly higher in SLE compared to controls $(p<0.01)$. Alexithymic patients presented increased values of BDI score and HAM-H score $(p<0.01$ and $p<0.05)$ and reduced Facit-Fatigue score $(p<0.05)$. We found increased values of Work missed due to health problems and Activity impairment in alexithymic SLE subjects $(p<0.05)$. SF-36 summary components PCS and MCS and SF-36 individual components did not significantly differ between alexithymic and non-alexithymic SLE. A greater proportion of alexithymic SLE patients presented fibromyalgia $(p<0.05)$, low scholastic education $(p<0.01)$, cognitive impairment according to MOCA test $(p<0.01)$ and mild-to-severe depression according to BDI $(p<0.01)$. We did not find differences among alexithymic and non alexithymic in MeS, obesity and physical inactivity prevalence. TAS-20 values positively correlated with BDI score $(p<0.001)$, HAM-H score $(p<0.01)$ and activity impairment score $(p<0.01)$ and negatively with Facit-Fatigue score $(p<0.01)$. In multiple linear regression, 
predictors of TAS-20 values were to be fibromyalgic and to have mild-to-severe depression according to BDI. In multiple logistic regression, alexithymia was significantly associated to $\mathrm{BDI}$ score (OR $1.2,95 \% \mathrm{Cl} 1.0-1.4)$ and inversely associated to cognitive impairment (OR $0.1,95 \% \mathrm{Cl} 0.02-0.8$ ).

Conclusions: SLE patients frequently present alexithymic tract. Alexithymia seems to be associated neither to disease feature, to disease course (activity and damage) and to SLE therapy, nor to HR-QoL expressed by SF-36. Nevertheless, alexithymia could be tightly related to QoL-associated factors as depression and fibromyalgia

Disclosure of Interest: None declared

DOI: 10.1136/annrheumdis-2017-eular.6679

\section{SAT0274 URINARY VITAMIN D-BINDING PROTEIN AS A BIOMARKER FOR LUPUS NEPHRITIS}

D.J. Go ${ }^{1,2}$, J.Y. Lee ${ }^{2}$, M.J. Kang ${ }^{2}$, I.A. Choi ${ }^{3}$, E.Y. Lee ${ }^{1}$, E.B. Lee ${ }^{1}$, E. Yi ${ }^{2}$, Y.W. Song ${ }^{1,2}$. 'Department of Internal Medicine, Seoul National University' Hospital; ${ }^{2}$ Department of Molecular Medicine and Biopharmaceutical Sciences, Graduate School of Convergence Science and Technology, and College of Medicine, Medical Research Institute, Seoul National University, Seoul; ${ }^{3}$ Department of Internal Medicine, Chungbuk National University Hospital, Cheongju, Korea, Republic Of

Background: Lupus nephritis (LN) is a major complication of systemic lupus erythematosus (SLE). However, conventional biomarkers for assessing renal disease activity are imperfect in predicting clinical outcomes associated with LN.

Objectives: The aim of this study is to identify urinary protein biomarkers that reliably reflect the disease activity or predict clinical outcomes.

Methods: A quantitative proteomic analysis, using liquid chromatography coupled with tandem mass spectrometry (LC-MS/MS), was performed to identify protein biomarker candidates that can differentiate between SLE patients with and without $\mathrm{LN}$. Selected biomarker candidates were further verified using urine samples from a larger cohort of SLE patients ( $n=121)$ by enzyme-linked immunosorbent assay (ELISA) to investigate their predictive values for $L N$ activity measure. Furthermore, association between urinary level of selected panel of potential biomarkers and prognosis of LN was assessed with a 4-year follow-up study of renal outcomes. Results: From proteomic assay, vitamin D binding protein (VDBP), transthyretin (TTR), retinol binding protein 4 (RBP4) and prostaglandin D synthase (PTGDS) were selected as candidates for quantification. These proteins were significantly elevated in SLE patients with LN, especially in patients with active LN $(n=21)$. Among them, VDBP well correlated with severity of proteinuria (rho $=0.661$, $P<0.001$ ) and renal SLE disease activity index (renal SLEDAI) ( $r$ ho $=0.520$, $\mathrm{P}<0.001$ ). In the 4 -year follow-up, VDBP was a significant risk factor (hazard ratio $9.627,95 \% \mathrm{Cl} 1.698$ to $54.571, \mathrm{P}=0.011$ ) for the development of proteinuric flare (randum urine protein/creatinine ratio $>1.0$ ) in SLE patients without proteinuria (random urine protein/creatinine ratio $<0.5)(n=100)$ after adjustments of multiple confounders.

Conclusions: Urinary VDBP correlated with proteinuria and renal SLEDAI, and predicted the development of proteinuria.

Disclosure of Interest: None declared

DOI: 10.1136/annrheumdis-2017-eular.4356

\section{SAT0275 COMPARISON OF CLINICAL AND SEROLOGICAL DIFFERENCES ACCORDING TO THE AUTOANTIBODY CLUSTER IN WOMEN WITH SYSTEMIC LUPUS ERYTHEMATOSUS: RESULTS FROM THE KOREAN LUPUS NETWORK (KORNET) REGISTRY}

\section{D.-J. Park, J.-H. Kang, J.-E. Kim, K.-E. Lee, S.-S. Lee. Chonnam National} University Medical School and Hospital, Gwangju, Korea, Republic Of

Objectives: Individual autoantibodies are associated with the clinical features in patients with systemic lupus erythematosus (SLE). However, few studies have investigated differences in disease presentation based on autoantibody profiles in Asian patients with SLE. This study evaluated autoantibody clusters and compared the clinical and serological presentation and clinical outcome in Korean SLE patients.

Methods: The Korean Lupus Network (KORNET) is a nationwide multicenter, hospital-based registry, set up to prospectively assess outcomes in Korean SLE patients. Of the 505 SLE patients enrolled in the KORNET registry from July 2014 to November 2015, the study group comprised 339 consecutive female SLE patients. Seven autoantibodies (anti-dsDNA, anti-Sm, anti-RNP, anti-Ro, anti-La, lupus anticoagulant (LAC), and anti-cardiolipin antibody [aCL]) were selected for cluster analysis using the K-means cluster analysis procedure.

Results: Three distinct autoantibody clusters were identified: cluster 1, antidsDNA and anti-Ro; cluster 2, anti-RNP; and cluster 3, anti-RNP, anti-Ro, and anti-La. Compared with patients in clusters $2(n=99)$ and $3(n=85)$, patients in cluster $1 \quad(n=155)$ had a shorter symptom duration before SLE diagnosis and higher incidence of biopsy-proven lupus nephritis. Patients in cluster 3 had a higher incidence of discoid rash, central nervous system involvement, lupus pancreatitis, pulmonary arterial hypertension, Raynaud's phenomenon, and premature gonadal failure. In addition, patients in cluster 3 had the lowest proportion of mean prednisolone $>7.5 \mathrm{mg} /$ day in the medication history.
Conclusions: Autoantibody clusters were associated with the clinical features in women with SLE. Clustering autoantibodies could be a valuable approach for differentiating between various clinical subsets of SLE, and may help to guide prediction of the subsequent clinical course and organ damage in these patients. Disclosure of Interest: None declared

DOI: 10.1136/annrheumdis-2017-eular.2361

\section{SAT0276 EYE TOXICITY IN PATIENTS WITH SYSTEMIC LUPUS ERITHEMATOSUS TREATED WITH ANTIMALARICS IN DOMINICAN REPUBLIC}

E. Rodríguez-Bautista ${ }^{1}$, Y. Cruz-Rojas ${ }^{1}$, I. Mercedes-Núñez ${ }^{1}$,

E. Tejada-Reyes ${ }^{1}$, N. Abreu-Arbaje ${ }^{2}$, M. Arbaje-Khoury ${ }^{2}$, V. Rosario ${ }^{1}$

R. Munoz-Louis ${ }^{1}$, R. Peña-Blanco ${ }^{3}$, T. Valdez-Lorie ${ }^{3}$, R. Alba-Fériz ${ }^{1}$.

${ }^{1}$ Rheumatology, Hospital Docente Padre Billini; ${ }^{2}$ Ophthalmology, Centro de

Oftalmología y Glaucoma; ${ }^{3}$ Rheumatology, Hospital Docente Universitario

Francisco Moscoso Puello, Santo Domingo, Dominican Republic

Background: Antimalarics are derivatives of quinine indicated in the treatment of autoimmune inflammatory diseases. The mechanism of antimalarial toxicity is unclear. It is hypothesized that toxicity is a result of drug binding to retinal pigmentary epithelium, damaging photoreceptors resulting in vision loss. Early retinal toxicity is asymptomatic with subtle alterations in foveal pigmentation generally not evident at routine ophthalmologic examination, progressively producing classic "bull's-eye" maculopathy, manifested as a decrease in central, color and night vision, and central scotoma. To prevent the sequelae of antimalarial use, sensitive tools are used to detect toxic maculopathy such as: campimetry, optical coherence tomography (OCT) and eye fundus.

Objectives: To evaluate ocular toxicity in patients with systemic lupus erythematosus treated with antimalarics.

Methods: Multicenter cross-sectional study, two rheumatology departments clinical records were analyzed from January 2016 to January 2017, with diagnosis of systemic lupus erythematosus according to ACR 1997 criteria, with $\geq 4$ years using antimalarial drugs. 298 patients were identified, 93 of them fulfilled inclusion criteria, and were evaluated by two retinologists performing OCT on each patient. Accumulated antimalarial doses were calculated and all variables were analyzed with SPSS software V.22.

Results: $97.8 \%$ were females, the mean age was $37.4 \pm 13$ years, $78.5 \%$ of the patients used $4 \mathrm{mg} / \mathrm{kg}$ of chloroquine (CQ) versus $21.5 \%$ took $6 \mathrm{mg} / \mathrm{kg}$ of hydroxychloroquine (HCQ), the mean use duration was $5.1 \pm 2$ years, $19.4 \%$ of patients had retinal pigment epithelium (RPE) changes suggesting maculopathy, of which, $15 \%$ used $C Q$ versus $4.35 \%$ with $\mathrm{HCQ}, 54.50 \%$ using $\mathrm{CQ}$ had a cumulative dose of 365 grams, $10.75 \%$ with HCQ had cumulative doses of 292 grams, and the mean for the cumulative dose of both antimalarials was 485 grams.

Conclusions: Previous studies have shown that the antimalarial toxicity rate are between $7.5 \%>13.1 \%$, in our population we observed that our patients had a higher toxicity rate associated with the use of $C Q$ compared to $H C Q$, and no association was found relevant with other variables. We understand that both, patients and physicians who manage this drug, should be educated about the need to maintain an adequate ophthalmologic control, due to the progression of retinopathy from 1 to 3 years after discontinuation of treatment. It is necessary to carry out prospective studies with a greater number of patients.

References:

[1] Block, J.A. (1998) Hydroxychloroquine and retinal safety. The Lancet 351(9105), 771-771.

[2] Battagliotti, C., Gentiletti, A., Pons-Estel, B. Lupus Eritematoso Sistémico, Aspectos Clínicos y Terapéuticos. $1^{\circ}$. Edición, 42, 515-531.

[3] Rosenbaum, J.T., Mount, G.R., et al. (2016). Avoiding Antimalarial Toxicity.Arthritis \& Rheumatology.

[4] Marmor MF, Melles RB. Hydroxychloroquine and the retina. JAMA 2015;313:847-8.

Disclosure of Interest: None declared

DOI: 10.1136/annrheumdis-2017-eular.4142

\section{SAT0277 COGNITIVE DYSFUNCTION IN PATIENTS WITH SYSTEMIC LUPUS ERYTHEMATOSUS IN DOMINICAN REPUBLIC}

E. Tejada-Reyes ${ }^{1}{ }^{\text {I. I. Mercedes-Nuñez }}{ }^{1}$, Y. Cruz-Rojas ${ }^{1}$,

E. Rodríguez-Bautista ${ }^{1}, \mathrm{~K}$. Polanco-Soriano ${ }^{2}$, M. Perdomo-Ramirez ${ }^{2}$,

V. Rosario ${ }^{1}$, R. Muñoz-Louis ${ }^{1}$, R. Peña-Blanco ${ }^{3}$, T. Valdez-Lorie ${ }^{3}$,

R. Alba-Fériz ${ }^{1} .{ }^{1}$ Rheumatology; ${ }^{2}$ Psychology, Hospital Docente Padre Billini;

${ }^{3}$ Rheumatology, Hospital Docente Universitario Dr. Francisco E. Moscoso Puello, Santo Domingo, Dominican Republic

Background: Cognitive dysfunction (CD) is a deficit of cognitive faculties including attention, memory, language, executive function and visuospatial processing. CD is the most frequent neuropsychiatric manifestation of SLE (55-80\%) 1 and this is 3 times higher in patients with Systemic Lupus Erythematosus (SLE) than in healthy subjects.2 This is not routinely evaluated because it requires a lot of time. Brief and simple questionnaires are needed to identify $C D$.

A study carried out by D'Amico et al. evaluated 21 SLE patients and all of them had CD.3 Pedraza et al. analyzed the MMSE score and Montreal Cognitive 